\title{
Some improved bounds on two energy-like invariants of some derived graphs*
}

\author{
Gui-Xian Tian ${ }^{a}$, Shu-Yu Cui ${ }^{b}$, \\ ${ }^{a}$ College of Mathematics, Physics and Information Engineering, \\ Zhejiang Normal University, Jinhua, Zhejiang, 321004, P.R. China \\ ${ }^{b}$ Xingzhi College, Zhejiang Normal University, Jinhua, Zhejiang, 321004, P.R. China
}

\begin{abstract}
Given a simple graph $G$, its Laplacian-energy-like invariant $L E L(G)$ and incidence energy $I E(G)$ are the sum of square root of its all Laplacian eigenvalues and signless Laplacian eigenvalues, respectively. Applying the Cauchy-Schwarz inequality and the Ozeki inequality, along with its refined version, we obtain some improved bounds on $L E L$ and $I E$ of the $\mathcal{R}$-graph and $\mathcal{Q}$-graph for a regular graph. Theoretical analysis indicates that these results improve some known results. In addition, some new lower bounds on $L E L$ and $I E$ of the line graph of a semiregular graph are also given.

AMS classification: 05C05; 05C12; 05C50

Keywords: regular graph; semiregular graph; incidence energy; $\mathcal{R}$ graph; $\mathcal{Q}$-graph; Laplacian-energy-like invariant
\end{abstract}

\section{Introduction}

We only consider finite simple graphs in this paper. Given a graph $G=(V, E)$ with vertex set $V=\left\{v_{1}, v_{2}, \ldots, v_{n}\right\}$ and edge set $E$, then $d_{i}=d_{G}\left(v_{i}\right)$ denotes the degree of $v_{i}$. If $d_{i}=r$ for any $i=1,2, \ldots, n$, then $G$ is called $r$-regular. If $G$ is a bipartite graph and $V=V_{1} \cup V_{2}$ is its bipartition, then $G$ is said to $\left(r_{1}, r_{2}\right)$ semiregular whenever each vertex in $V_{1}$ has degree $r_{1}$ and each vertex in $V_{2}$ has degree $r_{2}$. The adjacency matrix of $G$, denoted by $A(G)$, is a square matrix whose $(i, j)$-entry is one if $v_{i}$ and $v_{j}$ are adjacent in $G$ and zero otherwise. Let $D(G)$

\footnotetext{
${ }^{*}$ Corresponding author. E-mail: guixiantian@163.com (G.-X. Tian), cuishuyu@zjnu.cn (S.Y. Cui)
} 
be the degree diagonal matrix of $G$ with diagonal entries $d_{1}, d_{2}, \ldots, d_{n}$. Then $L(G)=D(G)-A(G)$ is called Laplacian matrix of $G$ and $Q(G)=D(G)+A(G)$ is called its signless Laplacian matrix.

Let $F$ be an $n \times n$ matrix associated to $G$, Then its characteristic polynomial $\psi(F ; x)=\operatorname{det}\left(x I_{n}-F\right)$ is called the $F$-polynomial of $G$, where $I_{n}$ is the identity matrix of order $n$. The zeros of $\psi(F ; x)$ is said to the $F$-eigenvalues of $G$. The set of all $F$-eigenvalues is called the $F$-spectrum of $G$. Specifically, if $F$ is one of the Laplacian matrix $L(G)$ and signless Laplacian matrix $Q(G)$ of $G$, then the corresponding spectrum are called respective $L$-spectrum and $Q$-spectrum. Throughout we denote the respective $L$-spectrum and $Q$-spectrum by $S p(L(G))=$ $\left\{\mu_{1}, \mu_{2}, \ldots, \mu_{n}\right\}$ and $S p(Q(G))=\left\{q_{1}, q_{2}, \ldots, q_{n}\right\}$, where $\mu_{1} \geq \mu_{2} \geq \cdots \geq \mu_{n}=0$ and $q_{1} \geq q_{2} \geq \cdots \geq q_{n} \geq 0$ are the eigenvalues of $L(G)$ and $Q(G)$, respectively. For more details about $L$-spectrum and $Q$-spectrum of $G$, readers may refer to [2, 3, 4, 15, 9, 21].

For the $L$-spectrum of $G$, Liu and Liu [18] put forward the concept of the Laplacian-energy-like invariant, that is,

$$
L E L(G)=\sum_{i=1}^{n-1} \sqrt{\mu_{i}}
$$

The motivation of this concept derived from the Laplacian energy [13], along with graph energy [16]. Recently, Stevanović et al. 25] pointed out that $L E L$ has become a newly molecular descriptor. For more details about the mathematical properties of $L E L$, readers may refer to [7, 12, 19, 20, 26, 30, 32] and the references therein.

In 2007, Nikiforov also extended the definition of graph energy to any matrix $M$ [22]. The energy of $M$ is defined to the sum of all singular values of $M$, denoted by $E(M)$. Motivated further by above concepts $E(M)$ and $L E L$, Jooyandeh et al. [15] gave the definition of incidence energy $I E(G)=E(B(G))$ of a graph $G$, where $B(G)$ is the incidence matrix of $G$. It is easy to see that

$$
I E(G)=E(B(G))=\sum_{i=1}^{n} \sqrt{q_{i}} .
$$

For more details about $I E$, see [10, 11, 15, 31] and the references therein.

In recent years, $L E L$ and $I E$ of some operations on graphs have attracted people's attention. For example, some sharp bounds about $L E L$ are obtained by Wang and Luo 26] for the line graph, subdivision graph and total graph of regular graphs. Pirzada et al.[24] also presented some new bounds about $L E L$ for the line graph of semiregular graphs, the para-line graph, $\mathcal{R}$-graph, $\mathcal{Q}$-graph 
of regular graphs. In addition, Gutman et al. 11] presented some sharp bounds for $I E$ of the line graph and iterated line graph of regular graphs. Wang et al. 27] gave some new bounds for $I E$ of the subdivision graph and total graph of regular graphs. Wang and Yang [28] also presented some upper bounds on IE for the line graph of semiregular graphs, the para-line graph of a regular graph. Recently, Chen et al. 1] obtained some new bounds for $L E L$ and $I E$ of the line graph, subdivision graph and total graph of regular graphs. They pointed out that these results improved some known bounds in [11, 26].

Motivated by above researches, this paper gives some new bounds for $L E L$ of the $\mathcal{R}$-graph, $\mathcal{Q}$-graph of regular graphs. Theoretical analysis indicates that these results improve some known results obtained by Pirzada et al. in [24]. We also obtain some new bounds for $I E$ of the $\mathcal{R}$-graph, $\mathcal{Q}$-graph of regular graphs. These results are a useful supplement for the existing results on some bounds of $L E L$ and $I E$ of related graph operations of regular graphs in [1. In addition, some new lower bounds are also presented on $L E L$ and $I E$ for the line graph of semiregular graphs.

\section{Preliminaries}

Some definitions of line graphs, $\mathcal{R}$-graph and $\mathcal{Q}$-graph are recalled in this section and some lemmas are listed, which shall be used in the following sections.

Recall that the line graph $\mathcal{L}(G)[3]$ of $G$ is the graph whose vertix set is the edge set of $G$, and two vertices in $\mathcal{L}(G)$ are adjacent wenther the corresponding edges in $G$ have exactly a common vertex. Given an $\left(r_{1}, r_{2}\right)$-semiregular graph $G$ of order $n$ with $m$ edges, the $L$-spectrum and $Q$-spectrum of $\mathcal{L}(G)$ are, respectively,

$$
S p(L(\mathcal{L}(G)))=\left\{\left(r_{1}+r_{2}\right)^{(m-n)}, r_{1}+r_{2}-\mu_{1}, \ldots, r_{1}+r_{2}-\mu_{n}\right\}[24]
$$

and

$$
S p(Q(\mathcal{L}(G)))=\left\{\left(r_{1}+r_{2}-4\right)^{(m-n)}, r_{1}+r_{2}-4+q_{1}, \ldots, r_{1}+r_{2}-4+q_{n}\right\}[28],
$$

where $a^{(b)}$ indicate that $a$ is repeated $b$ times, $\left\{\mu_{1}, \mu_{2}, \ldots, \mu_{n}\right\}$ and $\left\{q_{1}, q_{2}, \ldots, q_{n}\right\}$ are the $L$-spectrum and $Q$-spectrum of $G$, respectively.

The $\mathcal{R}$-graph [3] of $G$, denoted by $\mathcal{R}(G)$, is the graph derived from $G$ by adding a vertex $w_{i}$ corresponding to every edge $e_{i}=u v$ of $G$ and by connecting every vertex $w_{i}$ to the end vertices $u$ and $v$ of $e_{i}$. If $G$ is an $r$-regular graph of order $n$ with $m$ edges, then $L$-polynomial [8, 29] and $Q$-polynomial [17] of $\mathcal{R}(G)$ are, 
respectively,

$$
\psi(L(\mathcal{R}(G)), x)=x(x-2)^{m-n}(x-r-2)^{n} \prod_{i=1}^{n-1}\left[(x-2)\left(x-r-\mu_{i}\right)-2 r+\mu_{i}\right]
$$

and

$$
\psi(Q(\mathcal{R}(G)), x)=(x-2)^{m-n}\left(x^{2}-(2+3 r) x+4 r\right) \prod_{i=2}^{n}\left[(x-2)\left(x-r-q_{i}\right)-q_{i}\right] .
$$

From (5) and (6) , we obtain easily:

Lemma 2.1[8, 17] If $G$ is an r-regular graph of order $n$ with $m$ edges, then

(i) If the L-spectrum of $G$ is $S p(L(G))=\left\{\mu_{1} \ldots, \mu_{n}\right\}$, then the L-spectrum of $\mathcal{R}(G)$ is

$$
S p(L(\mathcal{R}(G)))=\left\{2^{(m-n)}, \frac{\left(r+2+\mu_{i}\right) \pm \sqrt{\left(r+2+\mu_{i}\right)^{2}-12 \mu_{i}}}{2}(i=1,2, \ldots, n)\right\} .
$$

(ii) If the $Q$-spectrum of $G$ is $S p(Q(G))=\left\{q_{1}, \ldots, q_{n}\right\}$, then the $Q$-spectrum of $\mathcal{R}(G)$ is

$$
S p(Q(\mathcal{R}(G)))=\left\{2^{(m-n)}, \frac{\left(r+2+q_{i}\right) \pm \sqrt{\left(r+2+q_{i}\right)^{2}-4\left(2 r+q_{i}\right)}}{2}(i=1,2, \ldots, n)\right\} .
$$

The $\mathcal{Q}$-graph 3 of $G$, denoted by $\mathcal{Q}(G)$, is the graph derived from $G$ by plugging a vertex $w_{i}$ to every edge $e_{i}=u v$ of $G$ and by adding a new edge between two new vertices whenever these new vertices lie on adjacent edges of $G$. If $G$ is an $r$-regular graph of order $n$ with $m$ edges, then $L$-polynomial [8, 29] and $Q$-polynomial [17] of $\mathcal{Q}(G)$ are, respectively,

$$
\psi(L(\mathcal{Q}(G)), x)=x(x-2 r-2)^{m-n}(x-r-2) \prod_{i=1}^{n-1}\left[(x-r)\left(x-2-\mu_{i}\right)-2 r+\mu_{i}\right]
$$

and

$$
\begin{aligned}
\psi(Q(\mathcal{Q}(G)), x)= & (x-2 r+2)^{m-n}[(x-r)(x-4 r+2)-2 r] \\
& \times \prod_{i=2}^{n}\left[(x-r)\left(x-2 r+2-q_{i}\right)-q_{i}\right] .
\end{aligned}
$$


From (7) and (8), one obtains easily:

Lemma 2.2[8, 17] If $G$ is an $r$-regular graph of order $n$ with $m$ edges, then

(i) If the L-spectrum of $G$ is $S p(L(G))=\left\{\mu_{1}, \ldots, \mu_{n}\right\}$, then the L-spectrum of $\mathcal{Q}(G)$ is

$$
S p(L(\mathcal{Q}(G)))=\left\{(2 r+2)^{(m-n)}, \frac{\left(r+2+\mu_{i}\right) \pm \sqrt{\left(r+2+\mu_{i}\right)^{2}-4 \mu_{i}(r+1)}}{2}(i=1,2, \ldots, n)\right\} .
$$

(ii) If the $Q$-spectrum of $G$ is $S p(Q(G))=\left\{q_{1}, \ldots, q_{n}\right\}$, then the $Q$-spectrum of $\mathcal{Q}(G)$ is

$$
S p(Q(\mathcal{Q}(G)))=\left\{(2 r-2)^{(m-n)}, \frac{\left(3 r-2+q_{i}\right) \pm \sqrt{\left(3 r-2+q_{i}\right)^{2}-4 r\left(2 r-2+q_{i}\right)+4 q_{i}}}{2}\right\},
$$

where $i=1, \ldots, n$.

Lemma 2.3[1] If $G$ is an $r$-regular graph of order $n$, then

$$
\frac{n r}{\sqrt{r+1}} \leq L E L(G) \leq \sqrt{r+1}+\sqrt{(n-2)(n r-r-1)}
$$

where both equalities hold iff $G$ is the complete graph $K_{n}$.

Lemma 2.4[6] If $G$ is any graph of order $n$, with at least one edge, then $\mu_{1}=$ $\mu_{2}=\cdots=\mu_{n-1}$ iff $G$ is the complete graph $K_{n}$.

The following lemma for $Q$-spectrum is analogous to above Lemma 2.4 for $L$-spectrum. By Theorem 3.6 in [2], one obtains easily

Lemma 2.5 If $G$ is a graph of order $n$, with at least one edge, then $q_{2}=q_{3}=$ $\cdots=q_{n}$ iff $G$ is the complete graph $K_{n}$.

The following lemma comes from [23], which is called the Ozeki's inequality.

Lemma 2.6[23] Let $\xi=\left(a_{1}, \ldots, a_{n}\right)$ and $\eta=\left(b_{1}, \ldots, b_{n}\right)$ be two positive $n$ tuples with $0<p \leq a_{i} \leq P$ and $0<q \leq b_{i} \leq Q$, where $i=1, \ldots, n$. Then

$$
\sum_{i=1}^{n} a_{i}^{2} \sum_{i=1}^{n} b_{i}^{2}-\left(\sum_{i=1}^{n} a_{i} b_{i}\right)^{2} \leq \frac{1}{4} n^{2}(P Q-p q)^{2} .
$$


It is a remarkable fact that a refinement of Ozeki's inequality was obtained by Izumino et al.[14] as follows: Let $\xi=\left(a_{1}, \ldots, a_{n}\right)$ and $\eta=\left(b_{1}, \ldots, b_{n}\right)$ be two $n$-tuples with $0 \leq p \leq a_{i} \leq P, 0 \leq q \leq b_{i} \leq Q$ and $P Q \neq 0$, where $i=1, \ldots, n$. Take $\alpha=p / P$ and $\beta=q / Q$. If $(1+\alpha)(1+\beta) \geq 2$, then (9) still holds.

Remark that if $G$ is 1-regular, then $G$ is isomorphic to $\frac{n}{2} K_{2}$. For avoiding the triviality, we always suppose that $r \geq 2$ for an $r$-regular graph. In addition, for an $\left(r_{1}, r_{2}\right)$-semiregular graph $G, G$ is isomorphic to $\frac{n}{3} P_{3}$ whenever $r_{1}+r_{2}=3$. Next we also suppose that $r_{1}+r_{2} \geq 4$ for an $\left(r_{1}, r_{2}\right)$-semiregular graph throughout this paper.

\section{The Laplacian-energy-like invariant}

In this section, we shall give some improved bounds for $L E L$ of $\mathcal{R}$-graph and $\mathcal{Q}$-graph of regular graphs, as well as for the line graph of semiregular graphs. Now we first consider $L E L$ of $\mathcal{R}$-graph of regular graphs.

Theorem 3.1 If $G$ is an r-regular graph of order $n$ with $m$ edges, then (i)

$$
L E L(\mathcal{R}(G)) \leq \frac{n(r-2)}{2} \sqrt{2}+\sqrt{r+2}+(n-1) \sqrt{r+2+\frac{n r}{n-1}+\frac{2 \sqrt{3}}{n-1} L E L(G)},
$$

where the equality holds in (10) iff $G$ is the complete graph $K_{n}$.

(ii)

$$
L E L(\mathcal{R}(G)) \geq \frac{n(r-2)}{2} \sqrt{2}+\sqrt{r+2}+(n-1) \sqrt{\frac{3}{4}(r+2)+\frac{n r}{n-1}+\frac{2 \sqrt{3}}{n-1} L E L(G)} .
$$

Proof. Suppose that $S p(L(G))=\left\{\mu_{1}, \mu_{2}, \ldots, \mu_{n}\right\}$ is the $L$-spectrum of $G$. Then from (11) and the (i) in Lemma 2.1, one gets

$$
\begin{aligned}
\operatorname{LEL}(\mathcal{R}(G))= & \sum_{i=1}^{n-1}\left(\sqrt{\frac{\left(r+2+\mu_{i}\right)+\sqrt{\left(r+2+\mu_{i}\right)^{2}-12 \mu_{i}}}{2}}\right)+\sum_{i=1}^{n-1}\left(\sqrt{\frac{\left(r+2+\mu_{i}\right)-\sqrt{\left(r+2+\mu_{i}\right)^{2}-12 \mu_{i}}}{2}}\right) \\
& +(m-n) \sqrt{2}+\sqrt{r+2} \\
= & \sum_{i=1}^{n-1} \sqrt{\left(\sqrt{\frac{\left(r+2+\mu_{i}\right)+\sqrt{\left(r+2+\mu_{i}\right)^{2}-12 \mu_{i}}}{2}}+\sqrt{\frac{\left(r+2+\mu_{i}\right)-\sqrt{\left(r+2+\mu_{i}\right)^{2}-12 \mu_{i}}}{2}}\right)^{2}} \\
& +(m-n) \sqrt{2}+\sqrt{r+2} \\
= & \sum_{i=1}^{n-1} \sqrt{r+2+\mu_{i}+2 \sqrt{3 \mu_{i}}}+(m-n) \sqrt{2}+\sqrt{r+2}
\end{aligned}
$$


Notice that $\sum_{i=1}^{n-1} \mu_{i}=2 m=n r$. Applying the Cauchy-Schwarz inequality, one obtains

$$
\begin{aligned}
L E L(\mathcal{R}(G)) & \leq \sqrt{(n-1) \sum_{i=1}^{n-1}\left(r+2+\mu_{i}+2 \sqrt{3 \mu_{i}}\right)}+(m-n) \sqrt{2}+\sqrt{r+2} \\
& =(n-1) \sqrt{r+2+\frac{n r}{n-1}+\frac{2 \sqrt{3}}{n-1} L E L(G)}+\frac{n(r-2)}{2} \sqrt{2}+\sqrt{r+2}
\end{aligned}
$$

where above equality holds iff $\mu_{1}=\mu_{2}=\cdots=\mu_{n-1}$. It follows from Lemma 2.4 that $G$ is the complete graph $K_{n}$. Hence the proof of the (i) is completed.

Now we will prove the (ii). Assume that $a_{i}=\sqrt{r+2+\mu_{i}+2 \sqrt{3 \mu_{i}}}$ and $b_{i}=1, i=1, \ldots, n-1$. Take $P=\sqrt{3 r+2+2 \sqrt{6 r}}, p=\sqrt{r+2}$ and $Q=q=1$. Note that $0 \leq \mu_{i} \leq 2 r$. Thus $0<p \leq a_{i} \leq P, 0<q \leq b_{i} \leq Q$ and

$$
(P Q-p q)^{2}=(\sqrt{3 r+2+2 \sqrt{6 r}}-\sqrt{r+2})^{2}=(\sqrt{3 r}+\sqrt{2}-\sqrt{r+2})^{2} \leq r+2 .
$$

By Lemma 2.6, we obtain

$$
\begin{aligned}
\sum_{i=1}^{n-1} \sqrt{r+2+\mu_{i}+2 \sqrt{3 \mu_{i}}} & \geq \sqrt{(n-1) \sum_{i=1}^{n-1}\left(r+2+\mu_{i}+2 \sqrt{3 \mu_{i}}\right)-\frac{1}{4}(n-1)^{2}(P Q-p q)^{2}} \\
& \geq(n-1) \sqrt{\frac{3}{4}(r+2)+\frac{n r}{n-1}+\frac{2 \sqrt{3}}{n-1} L E L(G) .}
\end{aligned}
$$

From (12), one obtains the required result (ii).

Corollary 3.2 If $G$ is an $r$-regular graph of order $n$ with $m$ edges, then (i)

$$
L E L(\mathcal{R}(G)) \leq \frac{n(r-2)}{2} \sqrt{2}+\sqrt{r+2}+(n-1) \sqrt{r+2+\frac{n r}{n-1}+\frac{2 \sqrt{3}(\sqrt{r+1}+\sqrt{(n-2)(n r-r-1)})}{n-1}},
$$

where above equality holds iff $G$ is the complete graph $K_{n}$.

(ii)

$$
L E L(\mathcal{R}(G))>\frac{n(r-2)}{2} \sqrt{2}+\sqrt{r+2}+(n-1) \sqrt{\frac{3}{4}(r+2)+\frac{n r}{n-1}+\frac{2 \sqrt{3}}{n-1} \frac{n r}{\sqrt{r+1}}}
$$

Proof. The (i) in Theorem 3.1 and Lemma 2.3 implies that the (i) follows. Again, from Lemma 2.3 and (11), one gets

$$
L E L(\mathcal{R}(G)) \geq \frac{n(r-2)}{2} \sqrt{2}+\sqrt{r+2}+(n-1) \sqrt{\frac{3}{4}(r+2)+\frac{n r}{n-1}+\frac{2 \sqrt{3}}{n-1} \frac{n r}{\sqrt{r+1}}} .
$$


Suppose that the equality in (13) holds. From Lemma 2.3, we have $G$ is the complete graph $K_{n}$. But for the complete graph $K_{n}$, the inequality (10) implies that the equality is false in (11). This completes the proof.

Remark 1 Given an $r$-regular graph $G$ of order $n$, Pirzada et al. 24] proved that

$$
\frac{n(r-2)}{2} \sqrt{2}+n \sqrt{r+2}<L E L(\mathcal{R}(G)) \leq \frac{n(r-2)}{2} \sqrt{2}+\sqrt{r+2}+(n-1)(\sqrt{3 r}+\sqrt{2}),
$$

where the equality on the right of (14) holds iff $G$ is the complete graph $K_{2}$. Notice that these bounds in Corollary 3.2 improve those in (14). In fact, for the upper bound, we have

$$
\begin{aligned}
\sqrt{r+2+\frac{n r}{n-1}+\frac{2 \sqrt{3}(\sqrt{r+1}+\sqrt{(n-2)(n r-r-1)})}{n-1}} & \leq \sqrt{r+2+\frac{n r}{n-1}+\frac{2 \sqrt{3} \sqrt{(n-1) n r}}{n-1}} \\
& =\sqrt{r+2+\frac{n r}{n-1}+2 \sqrt{3} \sqrt{\frac{n r}{n-1}}} \\
& \leq \sqrt{r+2+2 r+2 \sqrt{3} \sqrt{2 r}} \\
& =\sqrt{3 r}+\sqrt{2},
\end{aligned}
$$

which implies that the upper bound in Corollary 3.2 is an improvement on that in (14). For the lower bound, it is easy to see that

$$
\sqrt{\frac{3}{4}(r+2)+\frac{n r}{n-1}+\frac{2 \sqrt{3}}{n-1} \frac{n r}{\sqrt{r+1}}}>\sqrt{\frac{3}{4}(r+2)+r}>\sqrt{r+2} .
$$

Hence the lower bound in Corollary 3.2 is also an improvement on that in (14).

Next we will consider the Laplacian-energy-like invariant of $\mathcal{Q}$-graphs of a regular graph.

Theorem 3.3 If $G$ is an r-regular graph of order $n$ with $m$ edges, then (i)

$$
L E L(\mathcal{Q}(G)) \leq \frac{n(r-2)}{2} \sqrt{2 r+2}+\sqrt{r+2}+(n-1) \sqrt{r+2+\frac{n r}{n-1}+\frac{2 \sqrt{r+1}}{n-1} L E L(G)},
$$

where the equality holds in (15) iff $G$ is the complete graph $K_{n}$. (ii)

$$
L E L(\mathcal{Q}(G))>\frac{n(r-2)}{2} \sqrt{2 r+2}+\sqrt{r+2}+(n-1) \sqrt{r+2+\frac{n r}{n-1}+\frac{2 \sqrt{r+1}}{n-1} L E L(G)-\frac{3}{4} r} .
$$


Proof. Assume that $S p(L(G))=\left\{\mu_{1}, \ldots, \mu_{n}\right\}$ is $L$-spectrum of $G$. By (i) in Lemma 2.2 and (1), one has

$$
\begin{aligned}
L E L(\mathcal{Q}(G))= & \sum_{i=1}^{n-1}\left(\sqrt{\frac{\left(r+2+\mu_{i}\right)+\sqrt{\left(r+2+\mu_{i}\right)^{2}-4(r+1) \mu_{i}}}{2}}\right)+\sum_{i=1}^{n-1}\left(\sqrt{\frac{\left(r+2+\mu_{i}\right)-\sqrt{\left(r+2+\mu_{i}\right)^{2}-4(r+1) \mu_{i}}}{2}}\right) \\
& +(m-n) \sqrt{2 r+2}+\sqrt{r+2} \\
= & \sum_{i=1}^{n-1} \sqrt{\left(\sqrt{\frac{\left(r+2+\mu_{i}\right)+\sqrt{\left(r+2+\mu_{i}\right)^{2}-4(r+1) \mu_{i}}}{2}}+\sqrt{\frac{\left(r+2+\mu_{i}\right)-\sqrt{\left(r+2+\mu_{i}\right)^{2}-4(r+1) \mu_{i}}}{2}}\right)^{2}} \\
& +(m-n) \sqrt{2 r+2}+\sqrt{r+2} \\
= & \sum_{i=1}^{n-1} \sqrt{r+2+\mu_{i}+2 \sqrt{(r+1) \mu_{i}}}+(m-n) \sqrt{2 r+2}+\sqrt{r+2}
\end{aligned}
$$

Notice that $\sum_{i=1}^{n-1} \mu_{i}=2 m=n r$. Applying the Cauchy-Schwarz inequality, one obtains

$$
\begin{aligned}
\operatorname{LEL}(\mathcal{Q}(G)) & \leq \sqrt{(n-1) \sum_{i=1}^{n-1}\left(r+2+\mu_{i}+2 \sqrt{(r+1) \mu_{i}}\right)}+(m-n) \sqrt{2 r+2}+\sqrt{r+2} \\
& =(n-1) \sqrt{r+2+\frac{n r}{n-1}+\frac{2 \sqrt{r+1}}{n-1} L E L(G)}+\frac{n(r-2)}{2} \sqrt{2 r+2}+\sqrt{r+2},
\end{aligned}
$$

which implies that the (15) follows. Furthermore, it is easy to verify that the equality in (15) holds iff $\mu_{1}=\mu_{2}=\cdots=\mu_{n-1}$. By Lemma 2.4, $G$ is the complete graph $K_{n}$. The proof of (i) is completed.

Next we will prove (ii). Assume that $a_{i}=\sqrt{r+2+\mu_{i}+2 \sqrt{(r+1) \mu_{i}}}$ and $b_{i}=1$, where $i=1, \ldots, n-1$. Take $P=\sqrt{3 r+2+2 \sqrt{2 r(r+1)}}, p=\sqrt{r+2}$ and $Q=q=1$. Notice that $0 \leq \mu_{i} \leq 2 r$ and $P=\sqrt{3 r+2+2 \sqrt{2 r(r+1)}} \leq$ $\sqrt{7 r+2}$. Thus $0<p \leq a_{i} \leq P, 0<q \leq b_{i} \leq Q$ and

$$
\begin{aligned}
(P Q-p q)^{2} & =(\sqrt{3 r+2+2 \sqrt{2 r(r+1)}}-\sqrt{r+2})^{2} \\
& \leq(\sqrt{7 r+2}-\sqrt{r+2})^{2}=\frac{(6 r)^{2}}{8 r+4+2 \sqrt{(7 r+2)(r+2)}} \\
& <\frac{36 r^{2}}{(8+2 \sqrt{7}) r}<3 r .
\end{aligned}
$$


From (17) and Lemma 2.6, one has

$$
\begin{aligned}
\sum_{i=1}^{n-1} \sqrt{r+2+\mu_{i}+2 \sqrt{(r+1) \mu_{i}}} \\
\quad \geq \sqrt{(n-1) \sum_{i=1}^{n-1}\left(r+2+\mu_{i}+2 \sqrt{(r+1) \mu_{i}}\right)-\frac{1}{4}(n-1)^{2}(P Q-p q)^{2}} \\
\quad>(n-1) \sqrt{r+2+\frac{n r}{n-1}+\frac{2 \sqrt{r+1}}{n-1} L E L(G)-\frac{3}{4} r} .
\end{aligned}
$$

From (17), one obtains the required result (ii).

By Theorem 3.3, we obtain Corollary 3.4 immediately.

Corollary 3.4 If $G$ is an r-regular graph of order $n$ with $m$ edges, then (i)

$$
\begin{aligned}
\operatorname{LEL}(\mathcal{Q}(G)) \leq & (n-1) \sqrt{r+2+\frac{n r}{n-1}+\frac{2 \sqrt{r+1}(\sqrt{r+1}+\sqrt{(n-2)(n r-r-1)})}{n-1}} \\
& +\frac{n(r-2)}{2} \sqrt{2 r+2}+\sqrt{r+2}
\end{aligned}
$$

where the equality holds iff $G$ is the complete graph $K_{n}$.

(ii)

$$
L E L(\mathcal{Q}(G))>\frac{n(r-2)}{2} \sqrt{2 r+2}+\sqrt{r+2}+(n-1) \sqrt{\left(\frac{3 n}{n-1}+\frac{1}{4}\right) r+2} .
$$

Remark 2 Given an $r$-regular graph $G$, Pirzada et al.[24] proved that

$$
\frac{n(r-2)}{2} \sqrt{2 r+2}+n \sqrt{r+2}<\operatorname{LEL}(\mathcal{Q}(G)) \leq(n-1) \sqrt{r}+\sqrt{r+2}+\frac{(n r-2) \sqrt{2 r+2}}{2},
$$

where the equality on the right of (18) holds iff $G$ is the complete graph $K_{2}$. Notice that $\left(\frac{3 n}{n-1}+\frac{1}{4}\right) r>r$, then the lower bound in Corollary 3.4 is an improvement on that in (18). For the upper bound, one has

$$
\begin{aligned}
\sqrt{r+2+\frac{n r}{n-1}+\frac{2 \sqrt{r+1}(\sqrt{r+1}+\sqrt{(n-2)(n r-r-1)})}{n-1}} & \leq \sqrt{r+2+\frac{n r}{n-1}+\frac{2 \sqrt{r+1} \sqrt{(n-1) n r}}{n-1}} \\
& =\sqrt{r+2+\frac{n r}{n-1}+2 \sqrt{r+1} \sqrt{\frac{n r}{n-1}}} \\
& <\sqrt{r+2+2 r+2 \sqrt{r+1} \sqrt{2 r}} \\
& =\sqrt{2 r+2}+\sqrt{r} .
\end{aligned}
$$

Hence, the upper bound in Corollary 3.4 is also an improvement on that in (18). 
We finally consider the $L E L$ of line graph of an $\left(r_{1}, r_{2}\right)$-semiregular graph. Pirzada et al.24] presented the following an upper bound on $L E L$ of line graph $\mathcal{L}(G)$ for an $\left(r_{1}, r_{2}\right)$-semiregular graph $G$, that is,

$$
L E L(\mathcal{L}(G)) \leq\left(\frac{n r_{1} r_{2}}{r_{1}+r_{2}}-n+1\right) \sqrt{r_{1}+r_{2}}+(n-2) \sqrt{\frac{n-1}{n-2}\left(r_{1}+r_{2}\right)-\frac{2 n r_{1} r_{1}}{(n-2)\left(r_{1}+r_{2}\right)}} .
$$

Next we shall give a lower bound on $L E L$ of its line graph $\mathcal{L}(G)$.

Theorem 3.5 If $G$ is an $\left(r_{1}, r_{2}\right)$-semiregular graph of order $n$ with $m$ edges, then

$L E L(\mathcal{L}(G)) \geq\left(\frac{n r_{1} r_{2}}{r_{1}+r_{2}}-n+1\right) \sqrt{r_{1}+r_{2}}+(n-2) \sqrt{\frac{3 n-2}{4 n-8}\left(r_{1}+r_{2}\right)-\frac{2 n r_{1} r_{2}}{(n-2)\left(r_{1}+r_{2}\right)}}$.

Proof. Suppose that $S p(L(G))=\left\{\mu_{1}, \ldots, \mu_{n}\right\}$ is the $L$-spectrum of $G$. Notice that $\mu_{1}=r_{1}+r_{2}$ and $\mu_{n}=0$. Then from (11) and (3), one gets

$$
L E L(\mathcal{L}(G))=(m-n+1) \sqrt{r_{1}+r_{2}}+\sum_{i=2}^{n-1} \sqrt{r_{1}+r_{2}-\mu_{i}} .
$$

Now, assume that $a_{i}=\sqrt{r_{1}+r_{2}-\mu_{i}}$ and $b_{i}=1, i=2, \ldots, n-1$. Take $P=$ $\sqrt{r_{1}+r_{2}}, p=0$ and $Q=q=1$. Obviously, $0 \leq p \leq a_{i} \leq P, 0 \leq q \leq b_{i} \leq Q$, $P Q \neq 0$ and $(1+p / P)(1+q / Q) \geq 2$. From the refined version of Ozeki's inequality, we have

$$
\begin{aligned}
\operatorname{LEL}(\mathcal{L}(G)) & \geq(m-n+1) \sqrt{r_{1}+r_{2}}+\sqrt{(n-2) \sum_{i=2}^{n-1}\left(r_{1}+r_{2}-\mu_{i}\right)-\frac{1}{4}(n-2)^{2}\left(r_{1}+r_{2}\right)} \\
& =(m-n+1) \sqrt{r_{1}+r_{2}}+(n-2) \sqrt{r_{1}+r_{2}-\frac{2 m-\left(r_{1}+r_{2}\right)}{n-2}-\frac{1}{4}\left(r_{1}+r_{2}\right)} \\
& =(m-n+1) \sqrt{r_{1}+r_{2}}+(n-2) \sqrt{\frac{3 n-2}{4 n-8}\left(r_{1}+r_{2}\right)-\frac{2 m}{n-2}},
\end{aligned}
$$

which yields the required result as $m=n r_{1} r_{2} /\left(r_{1}+r_{2}\right)$.

\section{The incidence energy}

Now we shall give some new bounds for $I E$ of $\mathcal{R}$-graph and $\mathcal{Q}$-graph of regular graphs, as well as for the line graph of semiregular graphs. Now we first consider $I E$ of $\mathcal{R}$-graph of regular graphs. 
Theorem 4.1 If $G$ is an r-regular graph of order $n$ with $m$ edges, then (i)

$$
I E(\mathcal{R}(G)) \leq \frac{n(r-2)}{2} \sqrt{2}+\sqrt{3 r+2+4 \sqrt{r}}+(n-1) \sqrt{\frac{2 n-3}{n-1} r+2 \sqrt{\frac{3 n-4}{n-1} r}+2},
$$

where the equality holds iff $G$ is the complete graph $K_{n}$.

(ii)

$$
I E(\mathcal{R}(G))>\frac{n(r-2)}{2} \sqrt{2}+\sqrt{3 r+2+4 \sqrt{r}}+(n-1) \sqrt{\left(\frac{2 n-3}{n-1}-\frac{2-\sqrt{3}}{2}\right) r+2 \sqrt{\left(\frac{3 n-4}{n-1}-\frac{3-2 \sqrt{2}}{2}\right) r}+2 .}
$$

Proof. Assume that $S p(Q(G))=\left\{q_{1}, \ldots, q_{n}\right\}$ is the $Q$-spectrum of $G$. Notice that $q_{1}=2 r$ as $G$ is $r$-regular. Then from (2) and the (ii) in Lemma 2.1, we easily obtain, by a simple calculation,

$$
I E(\mathcal{R}(G))=\sum_{i=2}^{n} \sqrt{r+q_{i}+2+2 \sqrt{2 r+q_{i}}}+(m-n) \sqrt{2}+\sqrt{3 r+2+4 \sqrt{r}}
$$

Notice that $\sum_{i=2}^{n} q_{i}=2 m-2 r=(n-2) r$. Applying the Cauchy-Schwarz inequality, one obtains

$$
\begin{aligned}
\sum_{i=2}^{n} \sqrt{r+2+q_{i}+2 \sqrt{2 r+q_{i}}} & \leq \sqrt{(n-1) \sum_{i=2}^{n}\left(r+2+q_{i}+2 \sqrt{2 r+q_{i}}\right)} \\
& =(n-1) \sqrt{\frac{2 n-3}{n-1} r+2+\frac{2}{n-1} \sum_{i=2}^{n} \sqrt{2 r+q_{i}}} \\
& \leq(n-1) \sqrt{\frac{2 n-3}{n-1} r+2+\frac{2}{n-1} \sqrt{(n-1) \sum_{i=2}^{n}\left(2 r+q_{i}\right)}} \\
& =(n-1) \sqrt{\frac{2 n-3}{n-1} r+2+2 \sqrt{\frac{3 n-4}{n-1} r}},
\end{aligned}
$$

From (21), we obtain the desired upper bound (19). Moreover, above equality occurs iff $q_{1}=2 r$ and $q_{2}=q_{3}=\cdots=q_{n}$. Thus by Lemma 2.5, $G$ is the complete graph $K_{n}$. The proof of (i) is completed.

Next we will prove (ii). Assume that $a_{i}=\sqrt{r+2+q_{i}+2 \sqrt{2 r+q_{i}}}$ and $b_{i}=$ $1, i=2, \ldots, n$. Take $P=\sqrt{3 r+2+4 \sqrt{r}}, p=\sqrt{r+2+2 \sqrt{2 r}}$ and $q=Q=1$. Notice that $0 \leq q_{i} \leq 2 r$. Thus $0<p \leq a_{i} \leq P, 0<q \leq b_{i} \leq Q$. By a simple computation, one has

$$
(P Q-p q)^{2}=(\sqrt{3 r+2+4 \sqrt{r}}-\sqrt{r+2+2 \sqrt{2 r}})^{2}<(4-2 \sqrt{3}) r .
$$


Then by Lemma 2.6, one has

$$
\begin{aligned}
& \sum_{i=2}^{n} \sqrt{r+2+q_{i}+2 \sqrt{2 r+q_{i}}} \geq \sqrt{(n-1) \sum_{i=2}^{n}\left(r+2+q_{i}+2 \sqrt{2 r+q_{i}}\right)-\frac{1}{4}(n-1)^{2}(P Q-p q)^{2}} \\
&>(n-1) \sqrt{\frac{(2 n-3) r}{n-1}+2+\frac{2}{n-1} \sum_{i=2}^{n} \sqrt{2 r+q_{i}}-\frac{1}{4}(4-2 \sqrt{3}) r .}
\end{aligned}
$$

Similarly, assume that $a_{i}=\sqrt{2 r+q_{i}}$ and $b_{i}=1, i=2, \ldots, n$. Take $P=$ $2 \sqrt{r}, p=\sqrt{2 r}$ and $Q=q=1$. Notice that $0 \leq q_{i} \leq 2 r$. Thus $0<p \leq a_{i} \leq P$, $0<q \leq b_{i} \leq Q$. Again by Lemma 2.6, one has

$$
\begin{aligned}
\sum_{i=2}^{n} \sqrt{2 r+q_{i}} & \geq \sqrt{(n-1) \sum_{i=2}^{n}\left(2 r+q_{i}\right)-\frac{1}{4}(n-1)^{2}(2 \sqrt{r}-\sqrt{2 r})^{2}} \\
& =(n-1) \sqrt{\left(\frac{3 n-4}{n-1}-\frac{3-2 \sqrt{2}}{2}\right) r} .
\end{aligned}
$$

Hence,

$$
\sum_{i=2}^{n} \sqrt{r+2+q_{i}+2 \sqrt{2 r+q_{i}}}>(n-1) \sqrt{\left(\frac{2 n-3}{n-1}-\frac{2-\sqrt{3}}{2}\right) r+2 \sqrt{\left(\frac{3 n-4}{n-1}-\frac{3-2 \sqrt{2}}{2}\right) r}+2},
$$

which, along with (21), implies the required result (ii).

Next we consider $I E$ of $\mathcal{Q}$-graphs for regular graphs.

Theorem 4.2 If $G$ is an r-regular graph of order $n$ with $m$ edges, then (i)

$$
\begin{aligned}
I E(\mathcal{Q}(G)) \leq & \frac{n(r-2)}{2} \sqrt{2 r+2}+\sqrt{5 r-2+4 \sqrt{r(r-1)}} \\
& +(n-1) \sqrt{\frac{4 n-5}{n-1} r+2 \sqrt{\frac{3 n-4}{n-1} r(r-1)}-2},
\end{aligned}
$$

where the equality holds in (22) iff $G$ is the complete graph $K_{n}$.

(ii)

$$
\begin{aligned}
I E(\mathcal{Q}(G))> & \frac{n(r-2)}{2} \sqrt{2 r+2}+\sqrt{5 r-2+4 \sqrt{r(r-1)}} \\
& +(n-1) \sqrt{\left(\frac{4 n-5}{n-1}-\frac{1}{4}\right) r+2 \sqrt{\left(\frac{3 n-4}{n-1}-\frac{3-2 \sqrt{2}}{2}\right) r(r-1)}-2 .}
\end{aligned}
$$

Proof. Assume that $S p(Q(G))=\left\{q_{1}, \ldots, q_{n}\right\}$ is the $Q$-spectrum of $G$. Notice that $q_{1}=2 r$ as $G$ is $r$-regular. Then from (2) and the (ii) in Lemma 2.2, it is easy to see that, by a simple calculation,

$$
\begin{aligned}
I E(Q(G))= & \sum_{i=2}^{n} \sqrt{3 r+q_{i}-2+2 \sqrt{r\left(2 r+q_{i}-2\right)-q_{i}}} \\
& +(m-n) \sqrt{2 r-2}+\sqrt{5 r-2+4 \sqrt{r(r-1)}} .
\end{aligned}
$$


Notice that $\sum_{i=2}^{n} q_{i}=2 m-2 r=(n-2) r$. Applying the Cauchy-Schwarz inequality, one obtains

$$
\begin{aligned}
& \sum_{i=2}^{n} \sqrt{3 r+q_{i}-2+2 \sqrt{r\left(2 r-2+q_{i}\right)-q_{i}}} \\
& \leq \sqrt{(n-1) \sum_{i=2}^{n}\left(3 r+q_{i}-2+2 \sqrt{r\left(2 r-2+q_{i}\right)-q_{i}}\right)} \\
& =(n-1) \sqrt{\frac{4 n-5}{n-1} r-2+\frac{2}{n-1} \sum_{i=2}^{n} \sqrt{r\left(2 r-2+q_{i}\right)-q_{i}}} \\
& \leq(n-1) \sqrt{\frac{4 n-5}{n-1} r-2+\frac{2}{n-1} \sqrt{(n-1) \sum_{i=2}^{n}\left[r\left(2 r-2+q_{i}\right)-q_{i}\right]}} \\
& =(n-1) \sqrt{\frac{4 n-5}{n-1} r-2+2 \sqrt{\frac{3 n-4}{n-1} r(r-1)}},
\end{aligned}
$$

which, along with (24), implies the desired upper bound. Moreover, above equality occurs iff $q_{1}=2 r$ and $q_{2}=q_{3}=\cdots=q_{n}$. Thus from Lemma 2.5, $G$ is the complete graph $K_{n}$. The proof of (i) is completed.

Now we will prove (ii). Assume that $a_{i}=\sqrt{3 r-2+q_{i}+2 \sqrt{r\left(2 r-2+q_{i}\right)-q_{i}}}$ and $b_{i}=1$, where $i=2, \ldots, n$. Take $P=\sqrt{5 r-2+4 \sqrt{r(r-1)}}, p=\sqrt{3 r-2+2 \sqrt{2 r(r-1)}}$ and $Q=q=1$. Notice that $0 \leq q_{i} \leq 2 r$. Thus $0<p \leq a_{i} \leq P, 0<q \leq b_{i} \leq Q$. By a simple computation, one has

$$
(P Q-p q)^{2}=\left(\sqrt{5 r-2+4 \sqrt{r(r+1)}}-\sqrt{3 r-2+2 \sqrt{2 r(r-1)})^{2}}<r .\right.
$$

Then by Lemma 2.6, one has

$$
\begin{aligned}
& \sum_{i=2}^{n} \sqrt{3 r-2+q_{i}+2 \sqrt{r\left(2 r-2+q_{i}\right)-q_{i}}} \\
& \geq \sqrt{(n-1) \sum_{i=2}^{n}\left(3 r-2+q_{i}+2 \sqrt{r\left(2 r-2+q_{i}\right)-q_{i}}\right)-\frac{1}{4}(n-1)^{2}(P Q-p q)^{2}} \\
& >\sqrt{(n-1) \sum_{i=2}^{n}\left(3 r-2+q_{i}+2 \sqrt{r\left(2 r-2+q_{i}\right)-q_{i}}\right)-\frac{1}{4}(n-1)^{2} r} \\
& =(n-1) \sqrt{\left(\frac{4 n-5}{n-1}-\frac{1}{4}\right) r-2+\frac{2}{n-1} \sum_{i=2}^{n} \sqrt{r\left(2 r-2+q_{i}\right)-q_{i}} .}
\end{aligned}
$$

Similarly, suppose that $a_{i}=\sqrt{r\left(2 r-2+q_{i}\right)-q_{i}}$ and $b_{i}=1, i=2,3, \ldots, n$. Take $P=2 \sqrt{r(r-1)}, p=\sqrt{2 r(r-1)}$ and $Q=q=1$. Notice that $0 \leq q_{i} \leq 2 r$. 
Thus $0<p \leq a_{i} \leq P, 0<q \leq b_{i} \leq Q$. Again, from Lemma 2.6, one obtains

$$
\begin{aligned}
\sum_{i=2}^{n} \sqrt{r\left(2 r-2+q_{i}\right)-q_{i}} & \geq \sqrt{(n-1) \sum_{i=2}^{n}\left[r\left(2 r-2+q_{i}\right)-q_{i}\right]-\frac{1}{4}(n-1)^{2}(P Q-p q)^{2}} \\
& =(n-1) \sqrt{2 r(r-1)+\frac{n-2}{n-1} r(r-1)-\frac{1}{4}(2-\sqrt{2})^{2} r(r-1)} \\
& =(n-1) \sqrt{\left(\frac{3 n-4}{n-1}-\frac{3-2 \sqrt{2}}{2}\right) r(r-1)} .
\end{aligned}
$$

Hence,

$$
\sum_{i=2}^{n} \sqrt{3 r-2+q_{i}+2 \sqrt{r\left(2 r-2+q_{i}\right)-q_{i}}}>(n-1) \sqrt{\left(\frac{4 n-5}{n-1}-\frac{1}{4}\right) r+2 \sqrt{\left(\frac{3 n-4}{n-1}-\frac{3-2 \sqrt{2}}{2}\right) r(r-1)}-2} .
$$

From (24), one has the (ii) follows.

We finally consider the incidence energy of line graph for an $\left(r_{1}, r_{2}\right)$-semiregular graph. In [28], an upper bound on $I E$ of line graph $\mathcal{L}(G)$ for an $\left(r_{1}, r_{2}\right)$-semiregular graph $G$ was obtained as follows:

$$
\begin{aligned}
\operatorname{IE}(\mathcal{L}(G)) \leq & \left(\frac{n r_{1} r_{2}}{r_{1}+r_{2}}-n+1\right) \sqrt{r_{1}+r_{2}-4}+\sqrt{2\left(r_{1}+r_{2}\right)-4} \\
& +(n-2) \sqrt{\frac{n-3}{n-2}\left(r_{1}+r_{2}\right)+\frac{2 n r_{1} r_{2}}{(n-2)\left(r_{1}+r_{2}\right)}-4 .}
\end{aligned}
$$

Next one gives a lower bound for $I E$ of its line graph $\mathcal{L}(G)$.

Theorem 4.3 If $G$ is an $\left(r_{1}, r_{2}\right)$-semiregular graph with $n$ vertices and $m$ edges, then

$$
\begin{aligned}
I E(\mathcal{L}(G)) \geq & \left(\frac{n r_{1} r_{2}}{r_{1}+r_{2}}-n+1\right) \sqrt{r_{1}+r_{2}-4}+\sqrt{2\left(r_{1}+r_{2}\right)-4} \\
& +(n-2) \sqrt{\frac{3 n-10}{4 n-8}\left(r_{1}+r_{2}\right)+\frac{2 n r_{1} r_{2}}{(n-2)\left(r_{1}+r_{2}\right)}-4} .
\end{aligned}
$$

Proof. Suppose that $S p(Q(G))=\left\{q_{1}, \ldots, q_{n}\right\}$ is the $Q$-spectrum of $G$. Notice that $q_{1}=r_{1}+r_{2}$ and $q_{n}=0$. Then from (2) and (4), one gets

$$
I E(L(G))=(m-n+1) \sqrt{r_{1}+r_{2}-4}+\sqrt{2\left(r_{1}+r_{2}\right)-4}+\sum_{i=2}^{n-1} \sqrt{r_{1}+r_{2}-4+q_{i}} .
$$

Now, assume that $a_{i}=\sqrt{r_{1}+r_{2}-4+q_{i}}$ and $b_{i}=1, i=2, \ldots, n-1$. Take $P=$ $\sqrt{2\left(r_{1}+r_{2}\right)-4}, p=\sqrt{r_{1}+r_{2}-4}$ and $Q=q=1$. Obviously, $0 \leq p \leq a_{i} \leq P$, $0 \leq q \leq b_{i} \leq Q, P Q \neq 0$ and $(1+p / P)(1+q / Q) \geq 2$. By a simple computation, one has

$$
(P Q-p q)^{2}=\left(\sqrt{2\left(r_{1}+r_{2}\right)-4}-\sqrt{r_{1}+r_{2}-4}\right)^{2} \leq r_{1}+r_{2} .
$$


From the refined version of Ozeki's inequality, one has

$$
\begin{aligned}
\sum_{i=2}^{n-1} \sqrt{r_{1}+r_{2}-4+q_{i}} & \geq \sqrt{(n-2) \sum_{i=2}^{n-1}\left(r_{1}+r_{2}-4+q_{i}\right)-\frac{1}{4}(n-2)^{2}\left(r_{1}+r_{2}\right)} \\
& =(n-2) \sqrt{r_{1}+r_{2}-4+\frac{2 m-\left(r_{1}+r_{2}\right)}{n-2}-\frac{1}{4}\left(r_{1}+r_{2}\right)} \\
& =(n-2) \sqrt{\frac{3 n-10}{4 n-8}\left(r_{1}+r_{2}\right)+\frac{2 m}{n-2}-4}
\end{aligned}
$$

which, along with (25), implies the required result as $m=n r_{1} r_{2} /\left(r_{1}+r_{2}\right)$.

Acknowledgements This work was supported partly by NNSFC (11671053) and the Natural Science Foundation of Zhejiang Province, China (LY15A010011).

\section{References}

[1] X. Chen, Y. Hou, J. Li, On two energy-like invariants of line graphs and related graph operations, J. Inequal. Appl., 51 (2016) 1-15.

[2] D. Cvetković, New theorems for signless Laplacians eigenvalues, Bull. Acad. Serbe Sci. Arts, Cl. Sci. Math. Natur., Sci. Math., 137(33) (2008) 131-146.

[3] D. Cvetković, M. Doob, H. Sachs, Spectra of Graphs: Theory and Application, Academic press, New York, 1980.

[4] D. Cvetković, P. Rowinson, H. Simić, An introduction to the Theory of Graph Spectra, Cambridge University Press, Cambridge, 2010.

[5] D. Cvetković, P. Rowlinson, S.K. Simić, Signless Laplacians of finite graphs, Linear Algebra Appl., 423 (2007) 155-171.

[6] K. C. Das, A sharp upper bound for the number of spanning trees of a graph, Graphs Comb., 23 (2007) 625-632.

[7] K. C. Das, I. Gutman, A. S. Çevik, On the Laplacian-energy-like invariant, Linear Algebra Appl., 442 (2014) 58-68.

[8] A. Deng, A. Kelmans, J. Meng, Laplacian spectra of regular graph transformation, Discrete Appl. Math., 161 (2013) 118-133.

[9] R. Grone, R. Merris, V S. Sunder, The Laplacian Spectral of Graphs, SIAM J. Matrix Anal. Appl., 11 (1990) 218-239.

[10] I. Gutman, D. Kiani, M. Mirzakhah, On incidence energy of graphs, MATCH Commun. Math. Comput. Chem., 62 (2009) 573-580. 
[11] I. Gutman, D. Kiani, M. Mirzakhah, B. Zhou, On incidence energy of a graph, Linear Algebra Appl., 431 (2009) 1223-1233.

[12] I. Gutman, B. Zhou, B. Furtula, The Laplacian-energy-like invariant is an energy like invariant, MATCH Commun. Math. Comput. Chem., 64 (2010) 85-96.

[13] I. Gutman, B. Zhou, Laplacian energy of a graph, Linear Algebra Appl., 414 (2006) 29-37.

[14] S. Izumino, H. Mori, Y. Seo, On Ozeki's inequality, J. Inequal. Appl., 2 (1998) 235-253.

[15] M. R. Jooyandeh, D. Kiani, M. Mirzakhah, Incidence energy of a graph, MATCH Commun. Math. Comput. Chem., 62 (2009) 561-572.

[16] X. Li, Y. Shi, I. Gutman, Graph Energy, Springer, New York, 2012.

[17] J. Li, B. Zhou, Signless Laplacian characteristic polynomials of regular graph transformations, arXiv: 1303.5527v1 (2013).

[18] J. Liu, B. Liu, A Laplacian-energy-like invariant of a graph, MATCH Commun. Math. Comput. Chem., 59 (2008) 397-419.

[19] B. Liu, Y. Huang, Z. You, A survey on the Laplacian-energy-like invariant, MATCH Commun. Math. Comput. Chem., 66 (2011) 713-730.

[20] J.-B. Liu, X.-F. Pan, F.-T. Hu, F.-F. Hu, Asymptotic Laplacian-energy-like invariant of lattices, Appl. Math. Comput., 253 (2015) 205C214.

[21] R. Merris, Laplacian matrices of graphs: a survey, Linear Algebra Appl., 197-198 (1994) 143-176.

[22] V. Nikiforov, The energy of graphs and matrices., J. Math. Anal. Appl., 326 (2007) 1472-1475.

[23] N. Ozeki, On the estimation of the inequality by the maximum, J. Coll. Arts Sci., Chiba Univ. A., 5 (1968) 199-203.

[24] S. Pirzada, H. A. Ganie, I. Gutman, On Laplacian-energy-like invariant and Kirchhoff index, MATCH Commun. Math. Comput. Chem., 73 (2015) 41-59.

[25] D. Stevanović, A. Ilić, C. Onisor, M. Diudea, LEL-a newly designed molecular descriptor, Acta Chim. Slov., 56 (2009) 410-417.

[26] W. Wang, Y. Luo, On Laplacian-energy-like invariant of a graph, Linear Algebra Appl., 437 (2012) 713-721.

[27] W. Wang, Y. Luo, X. Gao, On incidence energy of some graphs, Ars Combin., 114 (2014) 427-436. 
[28] W. Wang, D. Yang, Bounds for incidence energy of some graphs, J. Appl. Math., article ID 757542 (2013).

[29] W. Wang, D. Yang, Y. Luo, The Laplacian polynomial and Kirchhoff index of graphs derived from regular graphs, Discrete Appl. Math., 161 (2013) 3063-3071.

[30] K. Xu, K. C. Das, Extremal Laplacian-energy-like invariant of graphs with given matching number, Electron J. Linear Algebra, 26 (2013) 131-140.

[31] B. Zhou, More upper bounds for the incidence energy, MATCH Commun. Math. Comput. Chem., 64 (2010) 123-128.

[32] B. X. Zhu, The Laplacian-energy like of graphs, Appl. Math. Lett., 24 (2011) 1604-1607. 